\section{Laudatio Prof. Dr. Karl-Heinz Deeg}

Mit der Verleihung der Ehrenmitgliedschaft „würdigt die DEGUM Personen, die sich große Verdienste bei der Förderung des medizinischen Ultraschalls erworben haben“ - für welchen Pädiater würde diese Anforderung besser gelten als für Professor Dr. med Karl-Heinz Deeg?

Gerne darf ich Ihnen zu dieser Frage meine Einschätzung geben:

Bereits während seiner Weiterbildung zum Pädiater und zum pädiatrischen Kardiologen an der Universitätsklinik in Erlangen zeigte Karl-Heinz Deeg sein Talent und seine Begeisterung für die ultraschallgestützte Bildgebung. Neben der Echokardiografie erlernte und studierte er den diagnostischen Ultraschall im Kindesalter. Im Jahre 1988 habilitierte er sich in Erlangen zum Thema „zerebrale Dopplersonografie beim Säugling“.

Sein wissenschaftliches Werk umfasst bislang 106 PubMed gelistete Publikationen, davon alleine 95 mit offensichtlichem Schwerpunkt „Sonografie“ und alleine 26 Arbeiten in „Ultraschall in der Medizin“. Da- neben ist Karl-Heinz Deeg Mitherausgeber mehrerer Monografien zum Thema Ultraschall oder Dopplersonografie, zuletzt der 4. Auflage von „Ultraschalldiagnostik in Pädiatrie und Kinderchirurgie“. Seine Arbeit zur Basilarisperfusion und plötzlichem Kindstod wurde 2010 mit dem DEGUMWissenschaftspreis ausgezeichnet.

Auch berufspolitisch hat sich Karl-Heinz Deeg für die Belange der Sektion und des Ultraschalls eingesetzt, so war er von 1992 - 2004 Vorsitzender der Sektion Pädiatrie und von 2011 - 2016 stellvertretender Sprecher der Sektion und verantwortlich für die Echokardiografie. Dass er natürlich Stufe III der DEGUM ist, zahlreiche Kurse und unzählige Vorträge abgehalten hat und im Beirat der „Ultraschall in der Medizin“ ist, sei hier nur am Rande erwähnt.

.... um nun auf meine anfangs gestellte Frage zu antworten: Professor Dr. Karl-Heinz Deeg ist der herausragende Vertreter des Ultraschalls in der Pädiatrie! Sein Beitrag zur Verbesserung des Wissenstandes und zur Ausbildung in unserem Fach ist nicht

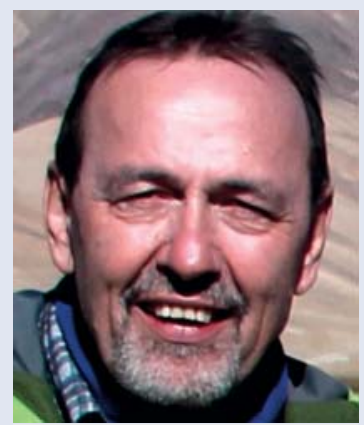

Prof. Dr. Karl-Heinz Deeg

hoch genug zu schätzen - und somit hat die DEGUM auf dem Dreiländertreffen in Leipzig nach einstimmigen Sektions-, Vorstands- und Mitgliedervotum völlig zu Recht beschlossen, Karl-Heinz Deeg die Ehrenmitgliedschaft der DEGUM zu verleihen.

Karl-Heinz, die Sektion Pädiatrie ist stolz auf Dich!

PD Dr. Udo Vester

Vorsitzender Sektion Pädiatrie DEGUM 